\title{
Right ventricular ejection fraction in postoperative cardiac surgery patients is independently associated with ICU morbidity and mortality
}

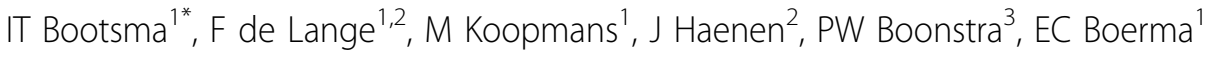 \\ From ESICM LIVES 2015 \\ Berlin, Germany. 3-7 October 2015
}

\section{Introduction}

Left ventricular heart failure is a well-known risk factor in cardiac surgery. However, data on the clinical relevance of right ventricular (RV) failure are limited. ${ }^{1}$

\section{Objectives}

To establish the prognostic implications of RV failure in a large series of post cardiac surgery patients.

\section{Methods}

We performed a single-centre retrospective analysis of all high risk cardiac surgery patients in a four year period. By protocol these patients were equipped with a pulmonary artery catheter (Vigilance ${ }^{\circledR}$, Baxter), enabling continuous RV ejection fraction (RVEF) measurements. RVEF was categorized into three subgroups: RVEF < $20 \%, 20-30 \%$ and $>30 \%$. Demographic data and hemodynamic variables were recorded. Primary outcome was predefined as the correlation between the average RVEF over the first 24 hours in the ICU and markers of morbidity.

\section{Results}

A total of 1115 patients were included. Patient characteristics are summarized in table 1. Patients with an RVEF < $20 \%$ had a significant longer duration of mechanical ventilation and lengths of stay in the ICU, higher ICU mortality, and increased use of inotropes and fluids. In a multivariate logistic regression model, RVEF appeared to be an independent risk factor for duration of mechanical ventilation, length of stay ICU, and ICU mortality.

\section{Conclusions}

A RVEF $<20 \%$ is independently associated with increased ICU mortality and morbidity in high risk postoperative cardiac surgery patients.

Table 1 Baseline characterstics.

\begin{tabular}{|c|c|c|c|c|}
\hline & RVEF $<20 \%(N=218)$ & RVEF $20-30 \%(N=750)$ & RVEF $>30 \%(N=147)$ & P-value \\
\hline Age (years) & $74[67-79]$ & $70[63-77]$ & $65[58-73]$ & $<0.001$ \\
\hline Diabetes (\%) & 18 & 21 & 16 & 0.40 \\
\hline COPD (\%) & 22 & 17 & 12 & 0.04 \\
\hline$\overline{\text { NYHA III or IV (\%) }}$ & 50 & 37 & 35 & 0.01 \\
\hline Poor LVEF (\%) & 21 & 12 & 7 & $<0.001$ \\
\hline Additive euroSCORE 1 & $8[6-10]$ & $7[5-9]$ & $6[4-8]$ & $<0.001$ \\
\hline Aortic cross-clamp (min) & $98[70-128]$ & $97[68-139]$ & $93[65-134]$ & 0.68 \\
\hline$\overline{C A B G}(\%)$ & 13 & 12 & 11 & 0.43 \\
\hline
\end{tabular}

Data are median [interquartile range] or percentage. RVEF right ventricular ejection fraction. COPD chronic obstructive pulmonary disease. euroSCORE European system for cardiac operative risk. NYHA New York heart association. LVEF left ventricular ejection fraction. CABG coronary-artery bypass grafting. 
Table 2 Outcome.

\begin{tabular}{|c|c|c|c|c|}
\hline & RVEF $<20 \%(N=218)$ & RVEF $20-30 \%(\mathrm{~N}=750)$ & RVEF > 30\% ( $N=147)$ & P-value \\
\hline Mechanical ventilation (hours) & $5,5[3,5-17,6]_{a}$ & $4,5[3,0-8,0]_{b}$ & $3,5[2,5-5,5]_{c}$ & $<0,001$ \\
\hline Length of stay ICU & $2[2-5]_{a}$ & $2[2-3]_{b}$ & $2[2-2]_{c}$ & $<0,001$ \\
\hline Survival ICU (\%) & $96 a$ & $99_{b}$ & $99 \mathrm{~b}$ & 0,01 \\
\hline Use of inotropic drugs (\%) & $75 a$ & $61_{b}$ & $48_{c}$ & $<0,001$ \\
\hline Fluid balance (litres) & $1,9[1,2-3,2]_{a}$ & $1,6[0,8-2,8]_{b}$ & $1,1[0,3-2,0]_{c}$ & $<0,001$ \\
\hline
\end{tabular}

Data are median [interquartile range] or percentage. RVEF right ventricular ejection fraction. ICU intensive care unit. Groups are significantly different when $\mathrm{p}<$ 0,05. Subscript letters: Different letters indicate a significant difference of $p<0,05$ between groups.

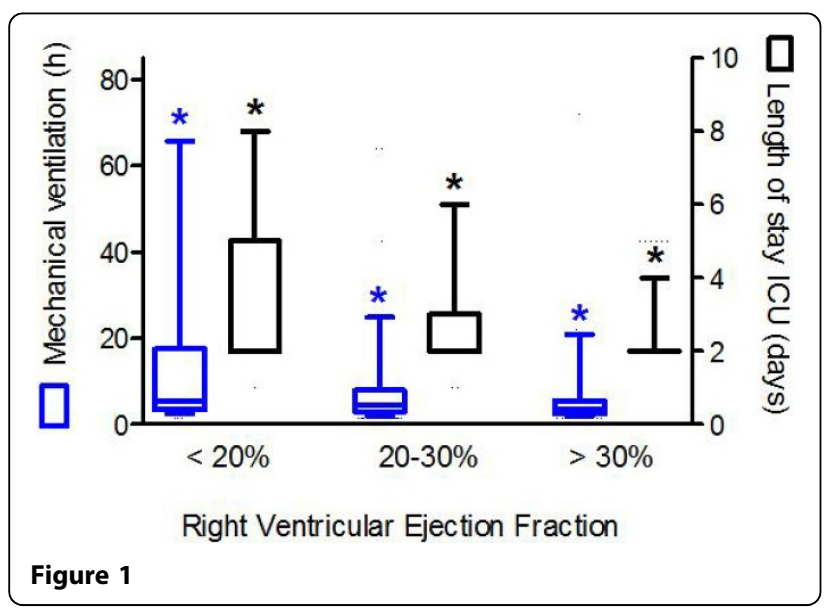

\section{Authors' details}

${ }^{1}$ Medical Centre Leeuwarden, Intensive Care Medicine, Leeuwarden, Netherlands. ${ }^{2}$ Medical Centre Leeuwarden, Cardiothoracic Anesthesiology, Leeuwarden, Netherlands. ${ }^{3}$ Medical Centre Leeuwarden, Cardiothoracic Surgery, Leeuwarden, Netherlands.

Published: 1 October 2015

\section{Reference}

1. Haddad F, et al: The Right Ventricle in Cardiac Surgery, a Perioperative Perspective: II. Pathophysiology, Clinical Importance, and Management. Anesthesia \& Analgesia 2009, 108:422-33.

doi:10.1186/2197-425X-3-S1-A112

Cite this article as: Bootsma et al:: Right ventricular ejection fraction in postoperative cardiac surgery patients is independently associated with ICU morbidity and mortality. Intensive Care Medicine Experimental 20153 (Suppl 1):A112.

\section{Submit your manuscript to a SpringerOpen ${ }^{\circ}$ journal and benefit from:}

- Convenient online submission

- Rigorous peer review

- Immediate publication on acceptance

- Open access: articles freely available online

- High visibility within the field

- Retaining the copyright to your article

Submit your next manuscript at $>$ springeropen.com 